\title{
A Multi-objective Placement of Phasor Measurement Units Considering Observability and Measurement Redundancy using Firefly Algorithm
}

\author{
K. Arul jeyaraj ${ }^{\dagger}$, V. Rajasekaran*, S. K. Nandha kumar* \\ and K. Chandrasekaran**
}

\begin{abstract}
This paper proposes a multi-objective optimal placement method of Phasor Measurement Units (PMUs) in large electric transmission systems. It is proposed for minimizing the number of PMUs for complete system observability and maximizing measurement redundancy of the buses, simultaneously. The measurement redundancy of the bus indicates that number of times a bus is able to monitor more than once by PMUs set. A high level of measurement redundancy can maximize the system observability and it is required for a reliable power system state estimation. Therefore, simultaneous optimizations of the two conflicting objectives are performed using a binary coded firefly algorithm. The complete observability of the power system is first prepared and then, single line loss contingency condition is added to the main model. The practical measurement limitation of PMUs is also considered. The efficiency of the proposed method is validated on IEEE 14, 30, 57 and 118 bus test systems and a real and large- scale Polish 2383 bus system. The valuable approach of firefly algorithm is demonstrated in finding the optimal number of PMUs and their locations by comparing its performance with earlier works.
\end{abstract}

Keywords: Firefly algorithm, Complete observability, Measurement redundancy, Optimal placement, Phasor measurement unit.

\section{Introduction}

\subsection{Analysis of recent trend of power system}

Power system restructuring, uneven expansion of generation and demand and other factors have disturbed and reduced the system stability level [1]. The unexpected rise of non-linear loads such as, air conditioners, heaters and motors leads to voltage instability problem. Consequently, recent trend and economical growth, power systems worldwide have become increasingly troubled with the voltage collapse problems [2]. The blackouts of 14th August 2003 in New York and July 30 and 31st 2012, in India, are few known examples, connected with voltage collapse problems. To avoid the occurrence of such blackouts, it is very important to consider the maximum loadability limit of the power systems [3]. In these situations, to ensure stable and proper operations, an exact measurement and monitoring of power system states are required. It is done by utilizing the Supervisory Control And Data Acquisition (SCADA) system, in which data

$\dagger \quad$ Corresponding Author: Dept. of Electrical and Electronic Engineering, PSNA College of Engineering \& Technology, Dindigul, Tamil nadu, India. (kajeyaraj@gmail.com)

* Dept. of Electrical and Electronic Engineering, PSNA College of Engineering \& Technology, Dindigul, Tamil nadu, India.

** Dept. of Electrical and Electronic Engineering, NIT Puducherry, India. (chansekaran23@gmail.com)

Received: January 14, 2014; Accepted: September 25, 2014 collected by Remote Terminal Units (RTUs) is not usually synchronized. In addition, SCADA could not supply any information about the dynamic state of the power system [4]. To beat this limitation of SCADA, Wide-Area Monitoring, Protection And Control (WAMPAC) system has been used, in which PMU is considered as a basic unit.

\subsection{Analysis of phasor measurement units}

A PMU is a power system monitoring device which was introduced in $1980 \mathrm{~s}$ that presents fast and smart communications. It plays a major role in a smart grid. The key characteristics of PMUs are measurements of voltage and current phasor that are time-stamped through a Global Positioning System, which supports to synchronize the data, taken from different locations in the widely dispersed power system. PMUs have better accuracy than SCADA system because of sampling the input waveforms with a higher rate [4]. The synchro phasor technology brings the opportunities to improve the real time monitoring, event analysis, protection and control of the power system [5]. Then, sampled data is transmitted through optical fiber to the phasor data concentrator (PDC), which is installed at the centralized location, for taking proper actions to remain a stable system. The PDC aligns the time of the data and presents it to the historian. It records the data for retrieval and post-dispatch analysis of any grid event. After getting the experience of main blackouts around the world, 
mainly on 14th August 2003, U.S.A blackout creates a new force for the implementation of Wide-Area Measurement Systems (WAMS) using PMUs. Currently, deciding the optimal number of PMUs and their locations in power systems has become main activity. It is one of the most important recommendations of U.S.A- Canada power system outage task force [6]. U.S.A, Brazil, China, Russia and other countries have already placed the PMUs and increased their potential benefits. The Government of India planned to place 1186 number of PMUs in their power system, gradually by $2014-15$, as a phase- I project [7].

\subsection{Analysis of optimal PMUs placement}

Optimal Placement of PMUs (OPP) method can be categorized as Conventional and Heuristic optimization method [8]. The most commonly, Integer Linear Programming (ILP) method is used to solve the OPP problem. In $[9,10]$, the authors used integer programming to determine the minimum number of PMUs. But, this method may experience from the difficulty of being trapped into local minima. One more disadvantage is that, starting from a first guess, it may direct to only one solution, while more than one solution may be present. Therefore, multiple objective problems cannot be handled by integer programming [11].

The stochastic search algorithms such as Genetic Algorithm (GA) [12], Immunity Genetic Algorithm (IGA) [13], Binary Particle Swarm Optimization (BPSO) [14] and Improved Tabu Search (ITS) [15] are presented to obtain the reliable OPP solutions. These techniques are found to be good for determining the global optimal solutions of PMUs placement and can be considered successful to a certain extent.

Since a new swarm based optimization techniques are observed as a promising one, the best location with less computation time is a tough task within the research part. In recent years, a new optimization method known as firefly algorithm was successfully developed by Yang to multimodal optimization [16].

In our previous work, firefly algorithm is tested and validated on power system optimization problems such as Economic Dispatch (ED) problem [17], Unit Commitment Problem (UCP) [18] and Multi-objective UCP (MUCP) [19]. In this context, an attempt is made to solve the Multiobjective OPP (MOPP) problem using a binary firefly algorithm.

\section{Proposed Work}

The aim of this paper is to prove the efficiency of firefly algorithm for solving the Multi-objective Optimal Placement of PMUs (MOPP) problem. The major work of this paper is that minimizing the number of PMUs for complete system observability and maximizing the measurement redundancy, simultaneously.

The remaining part of the paper is organized as follows. Section 3, presents the proposed MOPP problem formulations with their constraints. Section 4, provides an overview of firefly algorithm and discusses the implementation of the binary coded firefly algorithm to solve the MOPP problem. Section 5, presents the proposed method results of standard test systems under normal conditions and single line loss conditions. The PMUs measurement channel limitation is also considered. Finally, the conclusion is discussed in Section 6.

\section{MOPP Problem Formulation}

In this proposed method, the optimal solution is achieved, by considering the zero injection bus effect to minimize the number of PMUs for complete system observability as well as bus measurement redundancy constraint to maximize the system observability. The placement of PMUs on all buses permits the direct measurement of state of the power system. But, PMU placement on each bus is difficult to accomplish either due to high cost of PMUs or unavailability of communication facilities in some places. The fundamental rule of PMU placement is that, when a PMU is placed on a bus, it can measure the voltage phasor on that bus, as well as on adjacent buses, using the measured current phasor and the known transmission line impedance [11]. Obviously, the installation of PMU makes that bus directly observable and its adjacent buses indirectly observable, by applying the Ohm's law $[10,20,21]$. This implies that a system can be made observable with a minimum number of PMUs by using the zero injection bus effect. A high level of measurement redundancy can maximize the system observability and it is essential for a reliable power system state estimation. To evaluate the ability of the proposed a MOPP problem formulation, optimal PMU placement for the standard test systems is performed.

\subsection{Minimizing number of PMUs}

The objective function stands for PMUs installation in a system. The observability of a bus depends on the installation of PMU on that bus or one of its incident buses. If a bus is said to be topologically observable, when there is at least one measurement on that bus, either directly or indirectly. Minimizing the number of PMUs for achieving a complete observability of the power system can be expressed as follows:

$$
N_{p m u}=\operatorname{Min} \sum_{i=1}^{N} p_{i}
$$

It is subjected to the following constraints,

$$
M * P \geq I
$$




$$
\begin{aligned}
& c_{i, j}=\left\{\begin{array}{l}
1, \text { if } i=j ; \\
1, \text { if } i \text { and } j \text { are adjacent buses } ; \\
0, \text { otherwise; }
\end{array}\right. \\
& P=\left[p_{1} p_{2} \ldots p_{N}\right]^{T} \\
& p_{i}=\left\{\begin{array}{l}
1, \text { if a PMU is installed at bus } \mathrm{i} ; \\
0, \text { otherwise; }
\end{array}\right.
\end{aligned}
$$

where $N_{\text {pqu }}$ is the minimum number of PMUs required, $N$ is the total number of buses in a system, $p_{i}$ is a binary variable which defines the possibility of a PMU on a bus, $M$ is the connectivity matrix of the system with a size of $N^{*} N$, which consists of binary element $c_{i, j}, P$ is the optimal PMUs placement vector, $I$ is the observability vector with a size of $N^{*} 1$. It should be equal to one or greater than one for complete system observability.

\subsection{Minimizing number of PMUs without zero injection bus effect}

Zero injection bus is a bus, which doesn't have any generator or load. If zero injection bus effect is not considered in the Optimal PMU Placement (OPP) problem formulation, then each bus should be observed at least once, either directly or indirectly through a PMU, for complete observability of the system. Then, observability vector $(U)$ in Eq. (2) becomes unit vector, with a size of $N^{*} 1$.

$$
I=[11 \ldots 1]^{T}
$$

\subsection{Minimizing number of PMUs with zero injection bus effect}

In OPP problem, required number of PMUs can be further minimized by considering the zero injection bus effect. The detailed explanation can be found in [20]. When the zero injection bus effect is incorporated into the OPP problem, Eq. (2) can be rewritten as Eq. (7). It is considered with the base case OPP problem formulation and is given by Eqs. (7)-(10).

$$
\begin{gathered}
M * P \geq K \\
R_{j}=1 \forall j \notin B_{1} \cup B_{2} \ldots B_{N Z} \\
\sum_{k \in B_{z}} R_{k} \geq\left|A_{z}\right| \forall z \in S_{z} \\
B_{z}=A_{z} \cup\{z\}
\end{gathered}
$$

where, $z$ is a zero injection bus, $A_{z}$ is the set of buses adjacent to bus $z, B_{z}$ is the set of adjacent buses to $z$ and itself, $N Z$ is the total number of zero injection buses in a system, $S_{z}$ is the set of zero injection buses in a system, $K$ is the observability vector with a size of $N^{*} 1$, not a unit vector under zero injection bus consideration.

When adjacent buses of the zero injection bus are observable except a bus, the unobservable bus will be observed by applying the KCL at zero-injection bus (i.e., zero injection bus effect) [10, 22-24]. Therefore, it is not needed to monitor all the zero injection buses or one of them adjacent buses. Hence, it minimizes the number of PMUs required for complete observability of the system.

\subsection{Maximizing system observability}

When a PMU is located in a bus, it can provide the real time-synchronized measurement of voltage phasor on that bus and also current phasor, at the connected branches of the bus [24]. It is assumed that the PMUs have a sufficient number of channels to measure the current phasors of all transmission lines, incident to the bus, at which they are placed.

The OPP problem does not have a single solution using this basic objective function. The optimization algorithms may result the different sets of solutions with the same minimum number of PMUs. Hence, Complete System Observability Redundancy Index (CSORI) and measurement redundancy may be considered as constraints in solving the OPP problem. A bus is said to be observable, when there is at least one measurement on that bus either directly or indirectly, through a PMU.

For solving the OPP problem, CSORI is used to calculate the complete system observability by adding the Bus Observability Index (BOI) of all the buses in a system. The $B O I$ is maximized randomly while keeping the same minimum number of PMUs which is obtained in base case OPP as in [20].

$$
\begin{gathered}
\text { CSORI }=\sum_{i=1}^{N} \mathrm{BOI}_{i} \\
1 \leq \mathrm{BOI}_{i} \leq\left(\tau_{i}+1\right) \\
\operatorname{Max}\left(\mathrm{BOI}_{i}\right) \text { or } \operatorname{Min}\left(N-\mathrm{BOI}_{i}\right) \\
\sum_{i=1}^{N} p_{i}=N_{p m u}
\end{gathered}
$$

where, CSORI represents the complete system observability redundancy index, which indicates the level of system observability, $B O I_{i}$ represents the number of PMUs which can monitor the bus $i, \tau_{i}$ represents the maximum connectivity of the bus $i$. $B O I_{i}$ value always lies between one and the maximum connectivity of the bus $i\left(\tau_{i}\right)$, It is considered for maximizing the complete system observability in all cases.

\subsection{Formulation of multi-objective function}

Most of the power system optimization problem involves the simultaneous optimization of several objective functions. These objective functions are non-commensurable, competing and conflicting in nature. The practical multiobjective optimization problem is given as follows: 


$$
F(x)=\operatorname{Min}\left[F_{1}(x), F_{2}(x), \ldots F_{n}(x)\right]
$$

It is subjected to the following constraints,

$$
\begin{gathered}
h_{e}(x) \leq 0, e=1,2, \ldots \mathrm{E}_{n} \\
y_{m}(x)=0, m=1,2, \ldots \mathrm{IE}_{n}
\end{gathered}
$$

where, $F_{I}(x), F_{2}(x) \ldots F_{n}(x)$ are different objective functions, $E_{n}$ is the number of equality constraint, $I E_{n}$ is the number of inequality constraints.

Multi-objective optimization method has such conflicting objective functions that give rise to a set of optimal solutions instead of single solution, since no solution can be considered better than any other, with respect to all objective functions. In this paper, two conflicting objective functions are considered and formulated as a constrained multi-objective function, given as follows:

$$
\operatorname{Min}\left[N_{p m u},\left(N-B O I_{i}\right)\right]
$$

It is subjected to the above constraint Eqs. (2)-(14). In Eq. (18), minimizing the difference between total number of buses and bus observability index $\left(N-B O I_{i}\right)$ is nothing but maximizing the measurement redundancy of the buses.

\section{Overview of Firefly Algorithm}

Firefly algorithm is a novel nature-inspired algorithm, inspired by the social behavior of fireflies. By idealizing some of the flashing characteristics of fireflies, fireflyinspired algorithm was successfully presented by Xin-She Yang in 2008. Firefly-inspired algorithm use the following three idealized rules: First, all fireflies are unisex which means that they are attracted to other fireflies, regardless of their sex. Secondly, the degree of the attractiveness of a firefly is proportional to its brightness, thus for any two flashing fireflies, the less bright one will move towards the brighter one. More brightness means less distance between two fireflies. However, if any two flashing fireflies are having same brightness, then they move randomly. Finally, the brightness of a firefly is determined by the value of the objective function.

For a maximization problem, the brightness of each firefly is proportional to the value of the objective function. In case of a minimization problem, the brightness of each firefly is inversely proportional to the value of the objective function. The detailed explanation can be found in our articles [17-19].

In this section, binary firefly algorithm is implemented to determine the optimal number of PMUs and their locations for the MOPP problem in order to get the best compromise solution. The step-by-step procedure for the proposed method is as follows:

\subsection{Implementation}

Step 1: Specify network data including the zero injection buses in a system. Initialize the control parameters of the firefly algorithm and maximum generation in the termination process.

Step 2: Evaluate the system observability and measurement redundancy using Eqs. (1) and (13).

Step 3: Initialize the population

Randomly generate a population of initial solutions represented by binary values. Hence initialize randomly an initial population, $R=\left[Y_{1} ; Y_{2} \ldots Y_{m}\right]$ of $m$ solutions or fireflies in the multi-dimensional solution space, where $m$ represents the size of the population and each solution of $Y$ is represented by the $\mathrm{S}$-dimensional vector. Here $S$ is equal to $N$.

Step 4: Modify the firefly position

A firefly produces a modification of the position based on the brightness between the fireflies. The new position is determined by altering the value of any one of the $N$ parameters (old firefly position), selected randomly using Eq. (19) and keeping other parameters unchanged. For the optimal placement of PMU problem, $B_{p q}$ represents 0 or 1 .

$$
B_{p q}^{\prime}=B_{p q}+\beta(r) *\left(B_{p q}-B_{f q}\right)+\alpha\left(\text { rand }-\frac{1}{2}\right)
$$

where $p, f \in\{1,2 \ldots m\}$ and $q \in\{1,2 \ldots N\}$ are randomly chosen indices. Although $f$ is determined randomly, it has to be different from $p$. The second term in an Eq. (19) is due to attraction. The third term introduces randomization with ' $\alpha$ ' being the randomization parameter and "rand" is a random number generated uniformly distributed between 0 and 1. The attractiveness function $\beta$ can be any of the monotonically decreasing functions as given in Eq. (20).

$$
\beta(r)=\beta_{0} e^{-\gamma r} p q^{n}, n \geq 1
$$

where $\gamma$ is the absorption coefficient, $r$ is the distance between two fireflies, $\beta_{0}$ is the initial attractiveness of the fireflies and $n$ is a constant.

Step 5: Repair the strategy for constraint management

Whenever the firefly position in the algorithm is modified, check the constraints (2-14). If there is any violation in constraint, alter the bit to zero to uninstall the PMU, in the corresponding bus to overcome the violation.

Step 6: For each position of firefly, the above two objection functions are evaluated using Eqs. (1) and (13).

Step 8: Memorize the best solution achieved so far. Increment the generation count.

Step 9: Stop the process if the termination criteria are satisfied. Termination criteria used in this work is the specified maximum number of generations. Otherwise, go 
to step 4. The best fitness and the corresponding position of the brightest, retained in the memory at the end of the termination criteria, is selected as the optimum number of PMUs and their locations for the test systems.

\section{Case Studies and Discussion}

The proposed PMU placement method is performed using firefly algorithm. All the programs are developed using MATLAB 7.01. The system configuration is Pentium IV processor with $3.2 \mathrm{GHz}$ speed and 1GB RAM. Firstly, base case OPP problem formulations and their results are discussed. Then, single line loss contingency condition and PMUs measurement limitation is considered. The applicability and validity of the firefly algorithm for the proposed method have been tested on standard IEEE 14, 30,57 and 118 bus systems and a real and large- scale Polish 2383 bus system.

Table 1 shows the different cases of MOPP problems considered. Table 2 shows the specifications of test systems [3, 22, 23] and [25, 26]. In MOPP problem, maximizing measurement redundancy is considered randomly on the buses. The advantage of maximizing measurement redundancy is that a major portion of the system will remain observable, in case one of the PMUs fails.

\subsection{Parameter SETTINGS of firefly algorithm}

The four control parameters of firefly algorithm such

Table 1. Different cases of MOPP Problems

\begin{tabular}{c|l}
\hline Case & \multicolumn{1}{c}{ Description } \\
\hline 1 & $\begin{array}{l}\text { To validate firefly algorithm without zero injection bus effect } \\
\text { under normal conditions }\end{array}$ \\
\hline 2 & $\begin{array}{l}\text { To validate firefly algorithm with zero injection bus effect } \\
\text { under normal conditions }\end{array}$ \\
\hline 3 & $\begin{array}{l}\text { To validate firefly algorithm with zero injection bus effect } \\
\text { under single line loss }\end{array}$ \\
\hline 4 & $\begin{array}{l}\text { To validate firefly algorithm with zero injection bus effect } \\
\text { under measurement limitation }\end{array}$ \\
\hline
\end{tabular}

Table 2. Specifications of test systems [3, 22, 23] and $[25,26]$

\begin{tabular}{c|c|c|c|c}
\hline $\begin{array}{c}\text { IEEE } \\
\text { system }\end{array}$ & $\begin{array}{c}\text { No. of zero } \\
\text { injection } \\
\text { buses, }(Z)\end{array}$ & $\begin{array}{c}\text { Set of zero } \\
\text { injection buses, } \\
\left(S_{z}\right)\end{array}$ & $\begin{array}{c}\text { Bus with } \\
\text { maximum } \\
\text { number of } \\
\text { branches }\end{array}$ & $\begin{array}{c}\text { Max. no. } \\
\text { branches } \\
\text { Connected to } \\
\text { a bus, }(\tau)\end{array}$ \\
\hline 14 bus & 1 & 7 & 4 & 5 \\
\hline 30 bus & 6 & $6,9,22,25,27,28$ & 6 & 7 \\
\hline 57 bus & 15 & $\begin{array}{l}4,7,11,21,22, \\
24,26,34,36,37, \\
39,40,45,46,48\end{array}$ & 9,13 & 6 \\
\hline $\begin{array}{c}118 \\
\text { bus }\end{array}$ & 10 & $\begin{array}{l}5,9,30,37,38, \\
63,64,6871,81\end{array}$ & 49 & 12 \\
\hline $\begin{array}{c}2383 \\
\text { bus }\end{array}$ & 552 & $\begin{array}{l}\text { not reported due to } \\
\text { high number }\end{array}$ & $\begin{array}{l}7,1095,1426,1919, \\
1647,1920\end{array}$ \\
\hline
\end{tabular}

as $\beta_{o}, \gamma, n$ and firefly size are to be determined, before its implementation. The feasible boundary limits of the control parameters for firefly algorithm are adapted from [27] and are given in Table 3. The setting of these parameters optimally would yield better solutions in a lesser computational time. By default setting of the parameters taken initially, one of the parameters is varied and the other parameters are kept constant. It has been tested for each parameter taking several values within a boundary limit.

There are thirty trials for each setting and they are performed in order to achieve some statistical information about the average evolution. Based on the above guidelines, numerical analysis is carried out to select the best parameter values. For instance, the average evolution of IEEE 14 bus system using a firefly algorithm is given in Table 4.

Similarly, the best combination of control parameters of the firefly algorithm is determined for other test systems and is tabulated in Table 5.

\subsection{Case 1}

In this case, MOPP problem is solved using firefly

Table 3. Feasible boundary limits of control parameters of firefly algorithm

\begin{tabular}{c|c|c}
\hline Sl. no. & Control parameter & Limits \\
\hline 1 & Absorption coefficient, $\gamma$ & $0-10$ \\
\hline 2 & Initial attractiveness, $\beta_{o}$ & $0-1$ \\
\hline 3 & Constant, $n$ & $0.1-3$ \\
\hline 4 & $\begin{array}{c}\text { Population size } \\
\text { (Firefly size) }\end{array}$ & $10-200$ \\
\hline
\end{tabular}

Table 4. Parameter settings of firefly algorithm for IEEE 14 bus system

\begin{tabular}{c|c|c|c|c|c|c|c|c}
\hline$\gamma$ & $\begin{array}{c}\text { Objective } \\
\text { function }\end{array}$ & CSORI & $\beta o$ & $\begin{array}{c}\text { Objective } \\
\text { function }\end{array}$ & CSORI & $\begin{array}{c}\text { Firefly } \\
\text { size }\end{array}$ & $\begin{array}{c}\text { Objective } \\
\text { function }\end{array}$ & CSORI \\
\hline 0 & 6 & 24 & 0 & 6 & 27 & 10 & 7 & 32 \\
0.5 & 5 & 20 & 0.1 & 6 & 26 & 20 & 5 & 22 \\
1 & 5 & 19 & 0.2 & 6 & 28 & 30 & 6 & 25 \\
1.5 & 7 & 35 & 0.3 & 5 & 21 & 50 & 5 & 24 \\
2 & 6 & 18 & 0.4 & 9 & 37 & 100 & 6 & 28 \\
5 & 6 & 26 & 0.5 & 9 & 38 & & & \\
10 & 5 & 19 & 0.6 & 8 & 35 & & & \\
& & & 0.7 & 7 & 31 & & & \\
& & & 0.8 & 5 & 21 & & & \\
& & & 0.9 & 5 & 22 & & & \\
& & & 1 & 6 & 26 & & & \\
\hline
\end{tabular}

Table 5. Control parameters of firefly algorithm for test systems

\begin{tabular}{c|c|c|c|c|c}
\hline Control parameter & 14 bus & 30 bus & 57 bus & 118 bus & 2383 bus \\
\hline $\begin{array}{c}\text { Absorption } \\
\text { coefficient, } \gamma\end{array}$ & 0.5 & 0.5 & 1.5 & 1.5 & 1.9 \\
\hline $\begin{array}{c}\text { Initial } \\
\text { attractiveness, } \beta_{o}\end{array}$ & 0.9 & 0.6 & 0.7 & 0.7 & 0.65 \\
\hline Constant, $n$ & 1 & 1 & 1 & 1 & 1 \\
\hline $\begin{array}{c}\text { Population size } \\
\text { (Fireflies size) }\end{array}$ & 50 & 50 & 100 & 200 & 200 \\
\hline
\end{tabular}


Table 6. Different solutions for IEEE 14 Bus System-Case 1

\begin{tabular}{|c|c|c|c|c|c|c|c|c|c|c|c|c|c|c|c|c|}
\hline \multirow{2}{*}{$\begin{array}{l}\text { Locations } \\
\text { of PMUs }\end{array}$} & \multicolumn{14}{|c|}{ Number of PMUs are able to observe the bus, $(B O I)$} & \multirow{2}{*}{ CSORI } & \multirow{2}{*}{$\begin{array}{l}\text { No. buses observed } \\
\text { more than once }\end{array}$} \\
\hline & 1 & 2 & 3 & 4 & 5 & 6 & 7 & 8 & 9 & 10 & 11 & 12 & 13 & 14 & & \\
\hline $2,6,7,9$ & 1 & 1 & 1 & 3 & 2 & 1 & 2 & 1 & 2 & 1 & 1 & 1 & 1 & 1 & 19 & 4 \\
\hline $2,7,10,13$ & 1 & 1 & 1 & 2 & 1 & 1 & 1 & 1 & 2 & 1 & 1 & 1 & 1 & 1 & 16 & 2 \\
\hline $2,6,8,9$ & 1 & 1 & 1 & 2 & 1 & 1 & 2 & 1 & 1 & 1 & 1 & 1 & 1 & 1 & 16 & 3 \\
\hline $2,7,11,13$ & 1 & 1 & 1 & 2 & 1 & 2 & 1 & 1 & 1 & 1 & 1 & 1 & 1 & 1 & 16 & 2 \\
\hline $2,8,10,13$ & 1 & 1 & 1 & 1 & 1 & 1 & 1 & 1 & 1 & 1 & 1 & 1 & 1 & 1 & 14 & 0 \\
\hline
\end{tabular}

Table 7. Measurement redundancy analysis using proposed method- Case 1

\begin{tabular}{|c|c|c|c|c|c|c|c|c|c|c|c|c|c|c|c|}
\hline \multirow{2}{*}{$\begin{array}{l}\text { Locations } \\
\text { of PMUs }\end{array}$} & \multicolumn{14}{|c|}{ Measurement redundancy of the buses } & \multirow{2}{*}{$\begin{array}{l}\text { No. buses observed } \\
\text { more than once }\end{array}$} \\
\hline & 1 & 2 & 3 & 4 & 5 & 6 & 7 & 8 & 9 & 10 & 11 & 12 & 13 & 14 & \\
\hline $2,6,7,9$ & 0 & 0 & 0 & 2 & 1 & 0 & 1 & 0 & 1 & 0 & 0 & 0 & 0 & 0 & 4 \\
\hline $2,7,10,13$ & 0 & 0 & 0 & 1 & 0 & 0 & 0 & 0 & 1 & 0 & 0 & 0 & 0 & 0 & 2 \\
\hline $2,6,8,9$ & 0 & 0 & 0 & 1 & 1 & 0 & 1 & 0 & 0 & 0 & 0 & 0 & 0 & 0 & 3 \\
\hline $2,7,11,13$ & 0 & 0 & 0 & 1 & 0 & 1 & 0 & 0 & 0 & 0 & 0 & 0 & 0 & 0 & 2 \\
\hline $2,8,10,13$ & 0 & 0 & 0 & 0 & 0 & 0 & 0 & 0 & 0 & 0 & 0 & 0 & 0 & 0 & 0 \\
\hline
\end{tabular}

Table 8. Comparison of algorithms- Case 1

\begin{tabular}{c|c|c}
\hline Algorithm & Mean time (Seconds) & $\begin{array}{c}\text { Frequency of achieving a minimum number of PMUs as well as maximum measurement } \\
\text { redundancy of the buses }\end{array}$ \\
\hline GA & 120 & 11 \\
\hline PSO & 40 & 15 \\
\hline Firefly & 38 & 23 \\
\hline
\end{tabular}

algorithm for normal operating condition of the power system, not including the zero injection bus effect. It should be noted that the obtained results are equal to the optimal number reported in the available literatures [10], $[11,20,28]$ and [29]. It justifies that the proposed firefly algorithm can be able to produce a better quality solution for solving the MOPP problem.

\subsubsection{IEEE 14 Bus System}

\subsubsection{Bus observability analysis}

Table 6 shows the different solutions of IEEE 14 bus system. From Table 6 , it is observed that five different solutions are acquired from thirty trials. It is also clear that, the first PMUs set gives a maximum system observability of the system than other PMUs sets.

\subsubsection{Measurement redundancy analysis}

Measurement redundancy is defined as the number of times a bus is observed more than once by PMUs set. The maximizing bus measurement redundancy constraint has the benefit that a larger portion of the power system will remain observable, in case, one of the PMUs fails.

From Table 7 , it is proved that the locations of PMUs, i.e., 2, 6, 7 and 9 are more desirable to guarantee the complete system observability and also enhance it.

\subsubsection{Robustness and computation efficiency of firefly} algorithm

In order to compare the robustness and computation efficiency of the firefly algorithm, the OPP problem is solved for IEEE 14 bus system using standard GA and PSO algorithm. Therefore, the initial random generated population is kept same for GA, PSO and firefly algorithm.

The comparison of efficiency of the three algorithms for IEEE 14 bus system is given in Table 8 . It is concluded that the firefly algorithm can offer best solutions of OPP with less computation time than GA and PSO. It should be noted that the PMUs placement problem is an off-line procedure. Therefore, the execution time is not much important in solving the OPP problem.

Fig. 1 shows the distribution of optimal number of PMUs and their locations obtained from 30 trial runs. It should be noted that the frequency of achieving the minimum number of PMUs with maximum measurement redundancy value is high, in case of firefly algorithm, when compared with GA and PSO algorithms. It shows the superiority of firefly algorithm over other algorithms.

Fig. 2 shows the convergence characteristic of GA, PSO and firefly for IEEE 14 bus system. From Fig. 2, it is clear that the characteristic of firefly is steadily reaching the

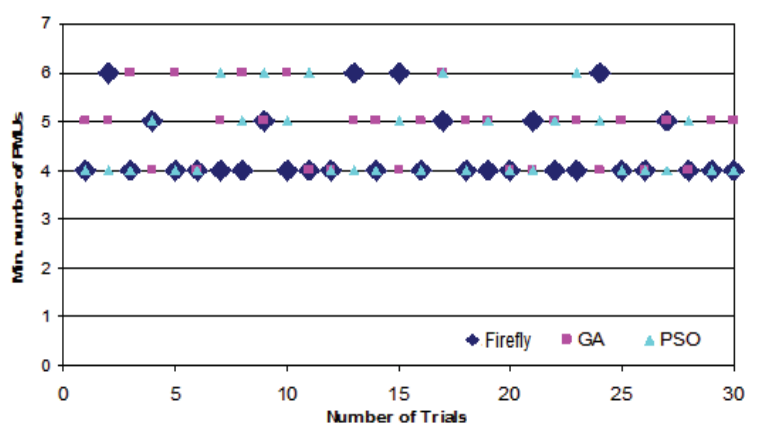

Fig. 1. Distribution of PMUs in IEEE14 bus system- Case1 
minimum value after a few iterations and produces better quality solutions. It can be concluded that firefly algorithm is computationally efficient in providing quality solutions obtained in minimum computation time at par with GA and PSO algorithms.

\subsubsection{IEEE 30 Bus System}

Table 9 shows the different solutions obtained from thirty trials run for IEEE 30 bus system. It is shown that numbers of buses are observed more than once is high in the first row as in Table 9 than any other sets. So, it is the best solution according to optimal PMU objective.

The PMU placement set of first PMUs set is similar to optimal solution in [4], fifth PMUs set is similar to [11], and the second PMUs set is similar to [30], but [30] has only 12 buses which are observed more than once so it is not good solution. The best OPP set is in the first row in Table 9 and have 14 buses which observed more than once even though system observability (CSORI) value is lower than value of second to fifth row PMUs set.

\subsubsection{IEEE 57 Bus System}

Table 10 shows the different solutions for IEEE 57 bus

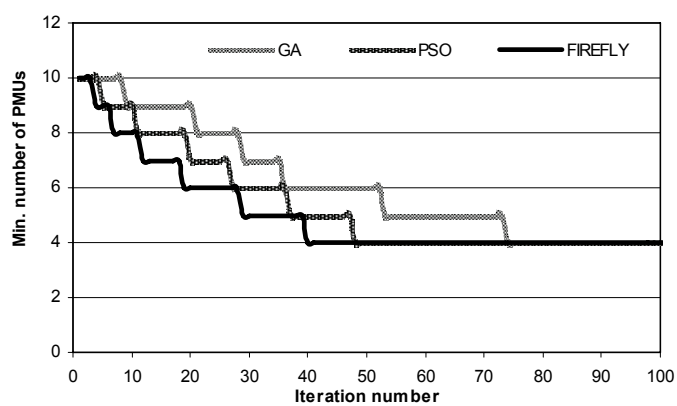

Fig. 2. MOPP problem convergence graph- IEEE 14 bus system- Case 1

Table 9. Different solutions for IEEE 30 bus system-Case 1

\begin{tabular}{c|c|c|c}
\hline Solution & Locations of PMUs, $P$ & CSORI & $\begin{array}{c}\text { No. buses observed } \\
\text { more than once }\end{array}$ \\
\hline 1 & $1,2,6,9,10,12,15,19,25,27$ & 50 & 14 \\
\hline 2 & $2,4,6,9,10,12,15,18,25,27$ & 52 & 12 \\
\hline 3 & $2,4,6,9,10,12,15,19,25,27$ & 52 & 13 \\
\hline 4 & $2,4,6,9,10,12,15,20,25,27$ & 52 & 12 \\
\hline 5 & $2,4,6,9,10,12,18,24,25,27$ & 51 & 12 \\
\hline 6 & $2,4,6,9,10,12,19,24,25,27$ & 51 & 12 \\
\hline 7 & $2,4,6,10,11,12,15,20,25,27$ & 50 & 12 \\
\hline 8 & $2,4,6,10,11,12,19,24,25,27$ & 49 & 12 \\
\hline 9 & $2,3,6,9,10,12,15,20,25,27$ & 48 & 11 \\
\hline 10 & $1,5,6,9,10,12,15,20,25,27$ & 48 & 13 \\
\hline 11 & $1,2,6,10,11,12,15,18,25,27$ & 48 & 13 \\
\hline 12 & $1,2,6,10,11,12,15,20,25,27$ & 48 & 13 \\
\hline 13 & $1,5,6,9,10,12,18,24,25,27$ & 47 & 12 \\
\hline 14 & $1,2,6,10,11,12,19,24,25,27$ & 47 & 12 \\
\hline 15 & $2,4,6,9,10,12,19,23,26,29$ & 46 & 8 \\
\hline 16 & $1,5,6,9,10,12,18,23,26,27$ & 44 & 9 \\
\hline 17 & $3,6,7,10,11,12,15,19,25,29$ & 44 & 11 \\
\hline
\end{tabular}

system. First two PMUs set are the best results according to the optimal PMU placement method. These two solutions have same minimum number of PMUs and maximum measurement redundancy of the buses.

The optimal PMUs placement set shown in second row in Table 10 is similar to the research work carried out in [4]. OPP solution of eighth PMUs set was the best result in [30], but actually it is not good solution, because it consists only 13 buses, which are observed more than once by this OPP set. IEEE 57 bus test systems consists of two best solutions set, as in first two rows in Table 10, and have 15 buses which observed more than once.

\subsubsection{IEEE 118 Bus system}

Table 11 shows the different PMUs placement sets

Table 10. Different solutions for IEEE 57 bus system-Case 1

\begin{tabular}{c|l|c|c}
\hline Solution & \multicolumn{1}{|c|}{ Locations of PMUs, $P$} & CSORI & $\begin{array}{c}\text { No. buses observed } \\
\text { more than once }\end{array}$ \\
\hline 1 & $\begin{array}{l}1,4,6,9,15,20,24,28,31,32, \\
36,38,41,46,51,53,57\end{array}$ & 72 & 15 \\
\hline 2 & $\begin{array}{l}1,4,6,9,15,20,24,25,28,32, \\
36,38,41,47,51,53,57\end{array}$ & 72 & 15 \\
\hline 3 & $\begin{array}{l}1,4,8,9,15,20,24,28,31,32, \\
36,38,39,41,47,50,53\end{array}$ & 71 & 13 \\
\hline 4 & $\begin{array}{l}1,6,9,15,19,22,25,26,29, \\
32,36,38,41,47,50,53,57\end{array}$ & 71 & 14 \\
\hline 5 & $\begin{array}{l}1,6,13,15,19,22,25,26,30, \\
32,36,38,41,47,51,54,57\end{array}$ & 71 & 13 \\
\hline 6 & $\begin{array}{l}1,4,7,9,15,19,22,25,25,27, \\
32,36,38,41,46,50,53,57\end{array}$ & 71 & 14 \\
\hline 7 & $\begin{array}{l}1,6,13,15,19,22,25,26,29, \\
32,36,38,41,47,51,54,57\end{array}$ & 71 & 13 \\
\hline 8 & $\begin{array}{l}1,4,7,9,15,20,24,25,27,32, \\
36,38,39,41,46,50,53\end{array}$ & 71 & 13 \\
\hline 9 & $\begin{array}{l}1,6,13,15,19,22,25,27,32, \\
36,38,41,47,51,52,55,57\end{array}$ & 70 & 12 \\
\hline 10 & $\begin{array}{l}1,6,9,15,18,21,24,28,31, \\
32,36,38,41,47,50,53,57\end{array}$ & 70 & 12 \\
\hline 11 & $\begin{array}{l}1,4,7,9,13,19,22,25,27,32, \\
36,41,44,47,50,53,57\end{array}$ & 69 & 11 \\
\hline 12 & $\begin{array}{l}1,4,9,10,20,22,25,26,29, \\
32,36,39,41,44,46,49,53\end{array}$ & 68 & 10 \\
\hline
\end{tabular}

Table 11. Different solutions for IEEE 118 bus systemCase 1

\begin{tabular}{c|c|c|c}
\hline Solution & Locations of PMUs, $P$ & CSORI & $\begin{array}{c}\text { No. buses observed } \\
\text { more than once }\end{array}$ \\
\hline \multirow{4}{*}{1} & $3,5,9,12,15,17,21,23,28$, & & \\
& $30,34,37,40,45,49,52,56$ & \multirow{2}{*}{164} & 39 \\
& $62,64,68,71,75,77,80,85$, & & \\
& $86,91,94,101,105,110,114$ & & \\
\hline \multirow{4}{*}{2} & $3,5,9,12,15,17,20,23,28$, & & \\
& $30,34,37,40,45,49,52,56$ & \multirow{2}{*}{164} & 38 \\
& $62,64,68,71,75,77,80,85$, & & \\
& $86,90,94,101,105,110,114$ & & \\
3 & $3,5,9,12,15,17,20,23,25$, & & \\
& $28,34,37,42,45,49,52,56$ & 162 & \\
& $62,64,68,71,75,77,80,85$, & & \\
& $86,90,94,102,105,110,115$ & & \\
\hline \multirow{4}{*}{4} & $2,5,9,12,15,17,21,25,28$, & & \\
& $34,37,42,45,49,53,56,62$ & \multirow{2}{*}{162} & \\
& $64,68,70,71,75,77,80,85$, & & \\
& $86,91,94,101,105,110,114$ & & \\
\hline
\end{tabular}


obtained from 30 trials run for IEEE 118 bus system. First row in Table 11 is similar to optimal solution given in [4] and has maximum measurement redundancy. It is clear that, the OPP consists of 39 buses which are observed more than once, since it is the best solution.

\subsubsection{Proposed Method Results}

The optimal locations are determined in such a way that which PMUs set offers the minimum number of PMUs and maximum measurement redundancy for complete system observability, under normal power system operating conditions. Here, maximizing bus measurement redundancy is considered, randomly on the buses. Therefore, complete system observability is improved. The results presented in Table 12, guarantee the complete observability of the system with minimum number of PMUs as well as maximum measurement redundancy of the buses, simultaneously.

\subsubsection{Comparative Analysis}

This proposed method demonstrates the minimum number of PMUs for complete power system observability, when the proposed result is compared with earlier works, as shown in Table 13. It should be noted that the obtained results are equal to the optimal number reported in the available literatures in [10,11, 20, 28, 29] but proposed method has higher measurement redundancy.

\subsection{Case 2}

In this case, MOPP problem is solved using firefly

Table 12. Optimal solutions of proposed method - Case 1

\begin{tabular}{|c|c|c|c|c|c|}
\hline $\begin{array}{c}\text { IEEE } \\
\text { system }\end{array}$ & $\begin{array}{l}\text { Min. } \\
\text { no. } \\
\text { PMUs }\end{array}$ & Locations of PMUs, $P$ & $\begin{array}{c}\text { PMU } \\
\text { buses in a } \\
\text { system, \% }\end{array}$ & CSORI & $\begin{array}{c}\text { No. buses } \\
\text { observed } \\
\text { more than } \\
\text { once }\end{array}$ \\
\hline 14 bus & 4 & $2,6,7,9$ & $28 \%$ & 19 & 4 \\
\hline 30 bus & 10 & $\begin{array}{l}1,2,6,9,10,12,15, \\
19,25,27\end{array}$ & $33 \%$ & 50 & 14 \\
\hline 57 bus & 17 & $\begin{array}{l}1,4,6,9,15,20,24, \\
28,31,32,36,38,41, \\
46,51,53,57\end{array}$ & $29 \%$ & 72 & 15 \\
\hline $\begin{array}{l}118 \\
\text { bus }\end{array}$ & 32 & $\begin{array}{l}3,5,9,12,15,17,21, \\
2328,30,34,37,40, \\
45,49,52,56,62,64, \\
68,71,75,77,80,85, \\
86,91,94,101,105, \\
110,114\end{array}$ & $27 \%$ & 164 & 39 \\
\hline
\end{tabular}

Table 13. The comparison of proposed results with earlier works-Case 1

\begin{tabular}{c|c|c|c|c}
\hline \multirow{2}{*}{ Method } & \multicolumn{4}{|c}{ Min. no. PMUs, $N_{p m u}$} \\
\cline { 2 - 5 } & 14 bus & 30 bus & 57 bus & 118 bus \\
\hline Proposed & 4 & 10 & 17 & 32 \\
\hline ILP, [10] & 4 & 10 & 17 & 32 \\
\hline Binary search, [11] & 4 & 10 & - & - \\
\hline ILP, [20] & 4 & - & 17 & 32 \\
\hline ILP, [28] & 4 & - & - & 32 \\
\hline ILP, [29] & 4 & - & 17 & 32 \\
\hline
\end{tabular}

algorithm, considering the zero injection bus effect. The optimal solution is obtained, after running 30 trials which gives the minimum number of PMUs and maximum measurement redundancy for normal operating conditions of the power system.

\subsubsection{IEEE 14 Bus System}

The proposed method offers the optimum solution for IEEE 14 bus system as in Table 14. It shows the efficiency of zero injection bus effect in the OPP problem. It further reduces the number of PMUs required for complete system observability.

\subsubsection{IEEE 30 Bus System}

Table 15 shows the different solutions of PMUs set for IEEE 30 bus system. It is shown that numbers of buses are observed more than once is high in the first row in Table 15 than any other sets. So, it is the best solution according to optimal PMU objective.

\subsubsection{IEEE 57 Bus System}

Table 16 shows the different solutions of PMUs set for IEEE 57 bus system. It is shown that numbers of buses are observed more than once is high in the first row in Table 16 than any other sets. So, it is the best solution according to optimal PMU objective.

Table 14. Optimal solution for IEEE 14 bus system- Case 2

\begin{tabular}{c|c|c|c|c}
\hline $\begin{array}{c}\text { Min. } \\
\text { no. of } \\
\text { PMUs }\end{array}$ & $\begin{array}{c}\text { Locations } \\
\text { of PMUs, } \\
P\end{array}$ & CSORI & $\begin{array}{c}\text { Average Measurement } \\
\text { redundancy } \\
\text { of the buses }\end{array}$ & $\begin{array}{c}\text { No. buses observed } \\
\text { more than once }\end{array}$ \\
\hline 3 & $2,6,9$ & 16 & 1.14 & 2 (buses 4 and 5) \\
\hline
\end{tabular}

Table 15. Different solutions for IEEE 30 bus system-Case 2

\begin{tabular}{|c|c|c|c|c|c|}
\hline Solution & $\begin{array}{l}\text { Min. no. } \\
\text { PMUs }\end{array}$ & $\begin{array}{c}\text { Locations } \\
\text { of PMUs, } \\
P\end{array}$ & CSORI & $\begin{array}{c}\text { Average } \\
\text { Measurement } \\
\text { redundancy of } \\
\text { the buses }\end{array}$ & $\begin{array}{c}\text { No. buses } \\
\text { observed } \\
\text { more than } \\
\text { once }\end{array}$ \\
\hline 1 & 7 & \begin{tabular}{|c|}
$2,4,10,12$ \\
$15,19,27$ \\
\end{tabular} & 41 & 1.36 & 8 \\
\hline 2 & 7 & $\begin{array}{c}2,4,10,12 \\
15,18,27\end{array}$ & 41 & 1.36 & 7 \\
\hline 3 & 7 & $\begin{array}{c}1,5,10,12 \\
18,24,27\end{array}$ & 37 & 1.23 & 3 \\
\hline 4 & 7 & \begin{tabular}{|c|}
$1,2,10,12$ \\
$18,24,27$ \\
\end{tabular} & 39 & 1.3 & 6 \\
\hline 5 & 7 & $\begin{array}{c}2,3,10,12, \\
19,24,27\end{array}$ & 36 & 1.2 & 5 \\
\hline 6 & 7 & $\begin{array}{c}3,7,10,12 \\
18,24,27\end{array}$ & 40 & 1.3 & 3 \\
\hline 7 & 7 & $\begin{array}{c}1,7,10,12 \\
19,24,27\end{array}$ & 41 & 1.36 & 4 \\
\hline 8 & 7 & $\begin{array}{c}3,5,10,12 \\
18,23,27\end{array}$ & 36 & 1.2 & 2 \\
\hline 9 & 7 & \begin{tabular}{|c|}
$1,5,10,12$ \\
$19,24,27$ \\
\end{tabular} & 37 & 1.23 & 4 \\
\hline 10 & 7 & $\begin{array}{c}1,5,10,12 \\
15,19,27 \\
\end{array}$ & 36 & 1.23 & 6 \\
\hline
\end{tabular}


Table 16. Different solutions for IEEE 57 bus system - Case 2

\begin{tabular}{c|c|c|c|c|c}
\hline Solution & Min. no. of PMUs & Locations of PMUs, $P$ & CSORI & $\begin{array}{c}\text { Average Measurement } \\
\text { redundancy of the buses }\end{array}$ & $\begin{array}{c}\text { No. buses observed more } \\
\text { than once }\end{array}$ \\
\hline 1 & 11 & $1,4,13,19,25,29,32,38,51,54,56$ & 64 & 1.12 \\
\hline 2 & 11 & $1,4,13,20,25,29,32,38,51,54,56$ & 63 & 1.105 \\
\hline 3 & 11 & $1,5,13,19,25,29,32,38,42,51,54$ & 61 & 1.07 \\
\hline 4 & 11 & $1,6,13,19,25,29,32,38,42,51,54$ & 61 & & \\
\hline
\end{tabular}

Table 17. Different solutions for IEEE 118 bus system - Case 2

\begin{tabular}{c|c|l|c|c|c}
\hline Sl.no. & Min. no. PMUs & \multicolumn{1}{|c|}{ Locations of PMUs, $P$} & CSORI & $\begin{array}{c}\text { Average bus measurement } \\
\text { redundancy }\end{array}$ & $\begin{array}{c}\text { No. buses observed } \\
\text { more than once }\end{array}$ \\
\hline 1 & 28 & $\begin{array}{l}3,8,11,12,17,21,27,31,32,34,37,40,45,49,52, \\
56,62,72,75,77,80,85,86,90,94,101,105,110\end{array}$ & 157 & 1.33 & 39 \\
\hline 2 & 28 & $\begin{array}{l}3,8,11,12,17,21,27,31,32,34,37,40,45,49,53, \\
56,62,72,75,77,80,85,86,90,94,102,105,110\end{array}$ & 156 & 1.32 & 38 \\
\hline 3 & 28 & $\begin{array}{l}3,8,11,12,19,22,27,31,32,34,37,40,45,49,53 \\
56,62,72,75,77,80,85,86,90,94,102,105,110\end{array}$ & 154 & 1.30 & 27 \\
\hline 4 & 28 & $\begin{array}{l}3,9,11,12,17,21,25,28,34,37,40,45,49,53,56, \\
62,72,75,77,80,85,86,90,94,102,105,110,114\end{array}$ & 149 & 1.26 & 31 \\
\hline 5 & 28 & $\begin{array}{l}3,9,11,12,17,21,25,29,34,37,41,45,49,53,56, \\
62,72,75,77,80,85,86,90,94,102,105,110,114\end{array}$ & 146 & 1.23 & 24 \\
\hline
\end{tabular}

Table 18. Optimal solutions of proposed method - Case 2

\begin{tabular}{c|c|c|c|c}
\hline $\begin{array}{c}\text { IEEE } \\
\text { system }\end{array}$ & Min. no. PMUs & Locations of PMUs, $P$ & $\begin{array}{c}\text { PMU buses in a system, } \\
\%\end{array}$ & $\begin{array}{c}\text { CSORI } \\
\text { more than once }\end{array}$ \\
\hline 14 bus & 3 & $2,6,9$ & $21 \%$ & 16 \\
\hline 30 bus & 7 & $2,4,10,12,15,19,27$ & $23 \%$ & 41 \\
\hline 57 bus & 11 & $1,4,13,19,25,29,32,38,51,54,56$ & $19 \%$ & 6 \\
\hline 118 bus & 28 & $\begin{array}{l}3,8,11,12,17,21,27,31,32,34,37,40,45,49,52,56, \\
62,72,75,77,80,85,86,90,94,101,105,110\end{array}$ & $24 \%$ & 7 \\
\hline
\end{tabular}

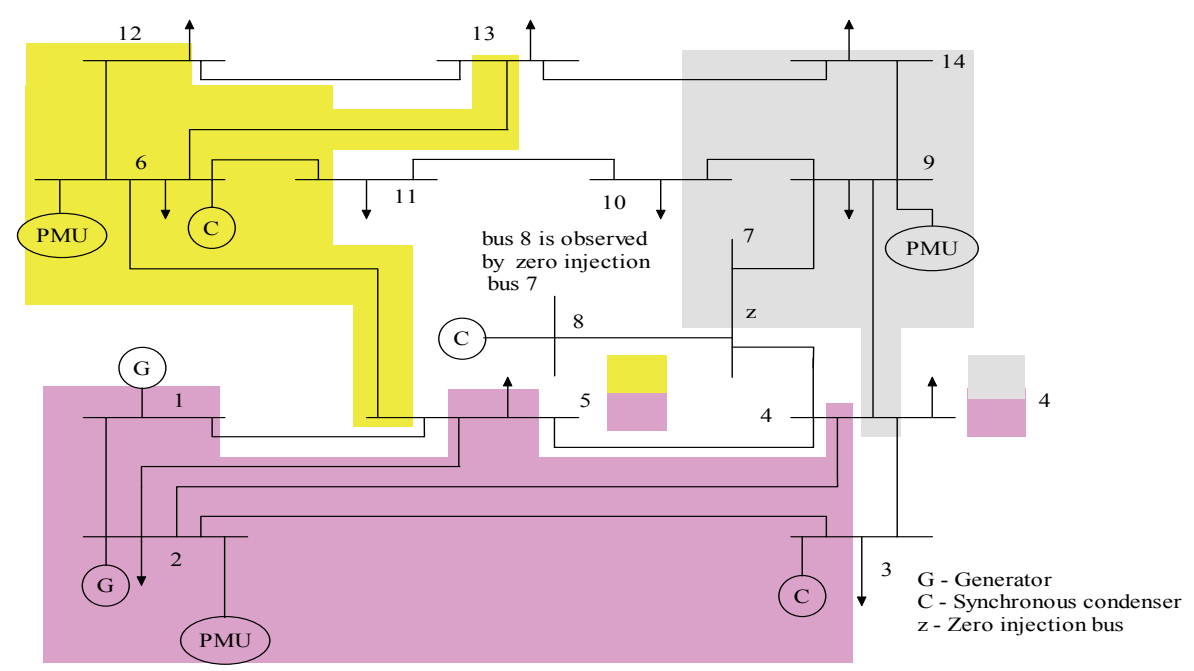

Fig. 3. IEEE 14 bus system with optimal PMU placement- Case 2

\subsubsection{IEEE 118 Bus System}

Table 17 shows the different solutions of PMUs set for IEEE 118 bus system. It is shown that numbers of buses are observed more than once is high in the first row in Table 17 than any other sets. So it is the best solution according to optimal PMU objective.

\subsubsection{Proposed Method Results}

The optimal locations are determined in such a way that which PMUs set offers the minimum number of PMUs and maximum measurement redundancy for complete system observability, under normal operating conditions, with zero injection bus consideration. Here, maximizing bus measurement redundancy is considered, randomly on the buses. Therefore, complete system observability is improved. The results presented as in Table 18, guarantee the complete observability of the system with minimum number of PMUs as well as maximum measurement redundancy of the buses, simultaneously. 
Table 19. The efficiency of the zero injection bus effectCase 2

\begin{tabular}{c|c|c|c}
\hline \multirow{2}{*}{ IEEE system } & \multicolumn{2}{|c|}{ Min. no. PMUs } & \multirow{2}{*}{$\begin{array}{c}\text { No. PMUs are } \\
\text { reduced }\end{array}$} \\
\cline { 2 - 3 } & Case 1 & Case 2 & 1 \\
\hline 14 bus & 4 & 3 & 3 \\
\hline 30 bus & 10 & 7 & 6 \\
\hline 57 bus & 17 & 11 & 4 \\
\hline 118 bus & 32 & 28 & \\
\hline
\end{tabular}

\subsubsection{Efficiency of zero injection bus effect}

It is clear that the total number of PMUs is considerably reduced for complete observability due to the addition of zero injection bus effect and shown in Table 19.

\subsubsection{Comparative analysis of minimum number of PMUs}

Table 20 shows the comparison of proposed method results with earlier methods. It shows that the proposed method is able to make a better MOPP solution. The optimal number of PMUs and their locations, observability area of each PMU, are shown as in Fig. 3 for IEEE 14 bus system.

\subsubsection{Comparative Analysis of Measurement Redundancy}

The proposed method proves that its efficiency in maximizing the number of buses observed more than once, when compared with earlier literatures, as shown in Table 21. It should be noted that the obtained results are equal to the optimal number reported in the available literatures but it is having higher measurement redundancy of the buses.

It is clear that, it can be concluded that characteristic of firefly is computationally efficient in providing quality solutions obtained in minimum computation time at par with BPSO as shown in Table 22.

\subsection{Case 3 - MOPP formulation for single line loss}

Single line loss is a most common type of fault, in the power systems. If two PMUs are observing a bus, then a related line loss will not affect the complete system observability. This can be modeled by modifying the

Table 20. The comparison of number of PMUs with earlier works-Case 2

\begin{tabular}{c|c|c|c|c|c|c|c|c|c}
\hline \multirow{2}{*}{ Method } & \multicolumn{3}{|c|}{ Min. no. PMUs required in IEEE systems } & \multirow{2}{*}{ Method } & \multicolumn{3}{|c}{ Min. no. PMUs required in IEEE systems } \\
\cline { 2 - 7 } \cline { 7 - 9 } & 14 bus & 30 bus & 57 bus & 118 bus & & 14 bus & 30 bus & 57 bus & 118 bus \\
\hline Proposed & 3 & 7 & 11 & 28 & BICA, [24] & 3 & 7 & 11 & 28 \\
\hline GA, [12] & 3 & 7 & 12 & 29 & RTS, [25] & 3 & 7 & 11 & 28 \\
\hline IGA, [13] & 3 & 7 & 11 & 29 & Dual search, [28] & 3 & - & - & 29 \\
\hline CLA, [21] & 3 & 7 & 11 & 29 & ILP, [29] & 3 & - & 14 & 29 \\
\hline ILP, [22] & 3 & 7 & 11 & 28 & PSO, [30] & 3 & 7 & 13 & 29 \\
\hline
\end{tabular}

Table 21. The comparison of observability and measurement redundancy results with earlier works-Case 2

\begin{tabular}{|c|c|c|c|c|c|c|c|c|}
\hline \multirow[b]{2}{*}{ Method } & \multicolumn{2}{|l|}{14 bus } & \multicolumn{2}{|l|}{30 bus } & \multicolumn{2}{|l|}{57 bus } & \multicolumn{2}{|l|}{118 bus } \\
\hline & $\begin{array}{c}\text { No. buses observed } \\
\text { more than once }\end{array}$ & CSORI & $\begin{array}{c}\text { No. buses observed } \\
\text { more than once }\end{array}$ & CSORI & $\begin{array}{c}\text { No. buses observed } \\
\text { more than once }\end{array}$ & CSORI & $\begin{array}{c}\text { No. buses observed } \\
\text { more than once }\end{array}$ & CSORI \\
\hline Proposed & 2 & 16 & 8 & 41 & 7 & 61 & 39 & 157 \\
\hline ILP, [20] & 1 & 15 & - & - & 4 & 61 & 31 & 152 \\
\hline IP, [22] & 2 & 16 & 4 & 41 & 2 & 59 & 31 & 156 \\
\hline BILP, [23] & 2 & 16 & 7 & 41 & 2 & 59 & 38 & 156 \\
\hline BICA, [24] & 2 & 16 & 8 & 41 & 2 & 59 & 38 & 156 \\
\hline
\end{tabular}

Table 22. The comparison of computation efficiency-Case 2

\begin{tabular}{c|c|c|c}
\hline \multirow{2}{*}{ Method } & \multicolumn{3}{|c}{ Average execution time in Seconds } \\
\cline { 2 - 4 } & 14 bus & 30 bus & 57 bus \\
\hline Proposed & 38 & 48 & 293 \\
\hline Modified BPSO, $[14]$ & 60 & 360 & 897 \\
\hline
\end{tabular}

Table 23. The optimal solutions of proposed method-Case 3

\begin{tabular}{|c|c|c|c|c|c|}
\hline $\begin{array}{l}\text { IEEE } \\
\text { system }\end{array}$ & $\begin{array}{l}\text { Min. no. } \\
\text { PMUs }\end{array}$ & Locations of PMUs, $P$ & PMU buses & CSORI & $\begin{array}{l}\text { Average measurement } \\
\text { redundancy }\end{array}$ \\
\hline 14 bus & 7 & $2,4,5,6,9,10,13$ & $50 \%$ & 34 & 2.43 \\
\hline 30 bus & 11 & $1,4,5,6,10,12,15,17,19,24,27$ & $43 \%$ & 52 & 1.73 \\
\hline 57 bus & 18 & $1,3,6,12,14,15,19,27,29,30,32,38,41,49,51,53,55,56$ & $33 \%$ & 92 & 1.61 \\
\hline 118 bus & 50 & $\begin{array}{l}1,6,10,11,12,15,17,21,23,25,27,29,32,34,40,42,44,46,49,51, \\
53,56,57,59,62,66,70,73,75,76,78,80,83,85,87,89,91,92,94,96, \\
100,102,105,106,109,111,112,115,116,117\end{array}$ & $45 \%$ & 252 & 2.13 \\
\hline
\end{tabular}


Table 24. The comparison of proposed results with earlier methods - Case 3

\begin{tabular}{c|c|c|c|c|c|c|c|c}
\hline \multirow{2}{*}{$\begin{array}{c}\text { IEEE } \\
\text { system }\end{array}$} & \multicolumn{2}{|c|}{ Proposed method } & \multicolumn{2}{c|}{ BPSO, [14] } & \multicolumn{2}{c|}{ BILP, [23] } & \multicolumn{2}{c}{ BICA, [24] } \\
\cline { 2 - 9 } & Min. no, PMUs & CSORI & Min. no, PMUs & CSORI & Min. no. PMUs & CSORI & Min. no. PMUs & CSORI \\
\hline 14 bus & 7 & 34 & 7 & - & 7 & 25 & 7 & 34 \\
\hline 30 bus & 11 & 52 & 15 & - & 13 & 50 & 11 & 52 \\
\hline 57 bus & 18 & 92 & 22 & - & 19 & 86 & 19 & 86 \\
\hline 118 bus & 50 & 252 & 62 & - & 53 & 242 & 53 & 242 \\
\hline
\end{tabular}

constraints given as,

$$
c_{i} * P \geq 2
$$

It is subjected to the above Eqs. (2) - (14). In Eq. (21), $c_{i}$, is the binary variable elements of the ith row of connectivity matrix $M$.

\subsubsection{Proposed method results}

The optimal number of PMUs and their locations for complete system observability under a single line loss condition are presented in Table 23. It can guarantee for satisfying our two objectives, simultaneously.

\subsubsection{Comparative analysis}

Table 24 presents a comparison of proposed results with other methods. In all cases the results of proposed method are either better than other methods or the same.

It is clear that, it can be concluded that characteristic of firefly is computationally efficient in providing quality solutions obtained in minimum computation time at par with BPSO as shown in Table 25.

Table 25. The comparison of computation efficiency-Case 3

\begin{tabular}{c|c|c|c|c}
\hline \multirow{2}{*}{ Method } & \multicolumn{4}{|c}{ Average execution time in Seconds } \\
\cline { 2 - 5 } & 14 bus & 30 bus & 57 bus & 118 bus \\
\hline Proposed & 41 & 60 & 308 & 957 \\
\hline Modified BPSO, [14] & 240 & 840 & 4800 & 13500 \\
\hline
\end{tabular}

\subsection{Simulation of real and large power system}

In order to examine the effectiveness of proposed approach in real and large-scale Polish 2383-bus power systems is studied. It includes 2896 lines and 552 zeroinjection buses [26]. The performance is assessed without considering the zero-injection bus effect (Case 1), the obtained optimal number of PMUs is 746 and including the zero injection bus effect (Case 2), the optimal results are shown as in Table 26. Here, 193 number of PMUs are reduced by considering the zero injection bus effect. The good quality of optimal solution in base case justifies the

Table 26. Optimal solutions- Polish 2383 bus system

\begin{tabular}{c|c|c|c}
\hline Min. no. PMUs & PMU buses, \% & CSORI & $\begin{array}{c}\text { Average measurement } \\
\text { redundancy }\end{array}$ \\
\hline 553 & $23 \%$ & 2788 & 1.17 \\
\hline
\end{tabular}

practical applicability of the proposed model.

\subsection{Case 4 - MOPP problem formulation for measure- ment limitation of PMUs}

In previous sections, it is assumed that PMUs can measure all the incident lines where it is placed. Since the number of PMU channels is limited in practice, a PMU installed on a bus could not measure the current phasors of all its incident lines. Thus, the aim of this section is to modify the formulation in Section 3.1 by considering the effect of PMUs measurement limitation. This modification can be done by changing the objective function as follows,

$$
N_{P M U}=\operatorname{Min} \sum_{i=1}^{N} w_{i j} * p_{i}, \forall \mathrm{j} \in N
$$

In addition the following constraints are added,

$$
\begin{gathered}
\sum_{i=1}^{N} c_{i, j} * w_{i j} \leq w_{i}^{\max }, \forall \mathbf{j} \in N \\
w_{i j} \leq p_{i}, \forall \mathbf{j} \in N
\end{gathered}
$$

where $c_{i, j}$ is a binary variable element in connectivity matrix $(M)$ as in Eq. (3), $\mathrm{w}_{\mathrm{ij}}$ is a binary variable which will be set to 1 to indicate that $j$ th bus is observable through the measured phasor current of the line $i-j$, by a PMU installed on $i$ th bus. Also, $w_{i}^{\max }$ is the maximum number of phasors which can be measured by a PMU installed on $i$ th bus. Hence, if it sets to 2, it implies that voltage phasor of the $i$ th bus in accompany with the current phasor of the only one its incident lines can be measured, as well. The number of PMUs required under measurement limitation is shown as in Table 27.

Table 27. Optimal number of PMUs considering measurement limitation of PMUs

\begin{tabular}{c|c|c|c|c}
\hline $\begin{array}{c}\text { PMUs channel limit } \\
\left(\mathrm{w}_{\mathrm{i}}^{\max }\right)\end{array}$ & $\begin{array}{c}\text { IEEE } \\
14 \text { bus }\end{array}$ & $\begin{array}{c}\text { IEEE } \\
30 \text { bus }\end{array}$ & $\begin{array}{c}\text { IEEE } \\
57 \text { bus }\end{array}$ & $\begin{array}{c}\text { IEEE 118 } \\
\text { bus }\end{array}$ \\
\hline 1 & 13 & 24 & 42 & 108 \\
\hline 2 & 7 & 12 & 21 & 54 \\
\hline 3 & 5 & 8 & 14 & 40 \\
\hline 4 & 4 & 8 & 13 & 36 \\
\hline 5 & 3 & 7 & 12 & 30 \\
\hline 6 & 3 & 7 & 11 & 29 \\
\hline 7 & 3 & 7 & 11 & 28 \\
\hline 8 & 3 & 7 & 11 & 28 \\
\hline 9 & 3 & 7 & 11 & 28 \\
\hline
\end{tabular}




\section{Conclusion}

This paper presented the application of binary coded firefly algorithm for solving the multi-objective OPP problems. The following points summarize the proposed work.

- The firefly algorithm, when applied to the OPP problem for normal operating conditions, is able to offer the minimum number of PMUs as well as maximum measurement redundancy of the systems, when compared with other techniques, reported in the earlier literatures.

- Multi-objective PMU placement problem has also been proposed for single line loss in the power systems and it is solved using firefly algorithm. From the comparative analysis, it is observed that the proposed methodology is efficient in determining the optimal number of PMUs and their locations under normal and contingency condition to maximize the observability and measurement redundancy of the systems, simultaneously. The practical limitation of PMUs measurement is also considered. The efficiency of the proposed method is validated on IEEE 14, 30, 57 and 118 bus test systems and real and large- scale Polish 2383 bus system.

\section{References}

[1] Mao, J. Yu, Z. Guo, "PMU placement and data processing in WAMS that complements SCADA", in IEEE Power Engineering Society General Meeting, vol. 1, pp. 780-783, June, 2005.

[2] Sood Yog Raj, "Evolutionary programming based optimal power flow and its validation for deregulated power system analysis", Int. Journal of Electrical Power and Energy Systems, vol. 29, pp. 65-75, 2007.

[3] P. Acharjee, "Identification of maximum loadability limit and weak buses using security constraint genetic algorithm", Electrical Power and Energy Systems, vol. 36, pp. 40-50, 2012.

[4] Saikat Chakrabarti, Elias Kyriakides, Demetrios G. Eliades, "Placement of Synchronized Measurements for Power System Observability", IEEE Trans. Power Delivery, vol. 24, no. 1, pp. 12-19, January, 2009.

[5] Jaime De La Ree, Virgilio Centeno, James S. Thorp, and A. G. Phadke, "Synchronized phasor measurement applications in power systems", IEEE Trans. Smart grid, vol. 1, no. 1, pp. 20-27, 2010.

[6] Final report on the august 14, "2003 black out in the United States and Canada: Causes and Recommendations April 5, U.S- Canada power system outage task force", pp.139- 140, 2004.

[7] A Report of Power Grid Corporation of India, Gurgaon, "Unified real time dynamic state measurement", Feb, 2012.

[8] Nikolaos M. Manousakis, George N. Korres, and
Pavlos S. Georgilakis, "Taxonomy of PMU placement methodologies", IEEE Trans. Power Systems, vol. 27, no. 2, pp. 1070-1077, 2012.

[9] B. Gou, "Generalized integer linear programming formulation for optimal PMU Placement", IEEE Trans. Power Syst., vol. 23, no. 3, pp. 1099-1104, 2008.

[10] B. Xu, Y. J. Yoon, and A. Abur, "Optimal placement and utilization of phasor measurements for state estimation", PSERC Publications, 2005.

[11] S. Chakrabarti, E. Kyriakides, “Optimal placement of phasor measurement units for power system observability”, IEEE Trans. Power Systems, vol.23, Aug, 2008, pp.1433-1440.

[12] F. J. Marin, F. Garcia-Lagos, G. Joya, and F. Sandoval, "Genetic algorithms for optimal placement of phasor measurement units in electric networks," Electron. Lett, vol. 39, no. 19, pp. 1403-1405, 2003.

[13] Farrokh Aminifar, Caro Lucas, Amin Khodaei, and Mahmud Fotuhi-Firuzabad, "Optimal placement of phasor measurement units using immunity genetic algorithm", IEEE Trans. Power Delivery, vol. 24, no. 3, pp. 1014-1020, 2009.

[14] M. Hajian, A.M. Ranjbar, T. Amraee, B. Mozafari, "Optimal placement of PMUs to maintain network observability using a modified BPSO algorithm", Int. Journal of Electrical Power and Energy Systems, vol. 33, pp. 28-34, Jan, 2011.

[15] Amany El-zonkoly, Samah El-safty, Rana Maher, "Optimal placement of PMUs using improved tabu search for complete observability and out-of-step prediction", Turkish Journal of Electrical Engg \& Computer Sciences, vol.21, pp.1376-1393, 2013.

[16] X.S. Yang, Firefly algorithms for multimodal optimization, in: Stochastic Algorithms: Foundations and Applications, Lecture Notes in Computer Sciences, vol. 5792, 2009, pp. 169-178.

[17] K. Chandrasekaran and Sishaj P. Simon, "Firefly algorithm for reliable/emission/economic dispatch multi objective problem", Int. Review of Electrical Engineering, vol. 7, no. 1, Part A, 2012, pp. 199-210.

[18] K. Chandrasekaran and Sishaj P. Simon, "Network and reliability constrained unit commitment problem using binary real coded firefly algorithm", Electrical Power and Energy Systems, vol. 43, no. 1, 2012, pp. 921-932.

[19] K. Chandrasekaran and Sishaj P. Simon, "Optimal deviation based firefly algorithm tuned fuzzy design for multi-objective UCP”, IEEE Trans. Power Systems, vol. 28 , no. 1,2013 , pp. 460- 471 .

[20] Dua, S. Dambhare, R.K. Gajbhiye and S.A. Soman, "Optimal multistage scheduling of PMU place-ment: An ILP approach", IEEE Trans. Power Delivery, vol. 23, no. 4, pp. 1812-1820. Oct, 2006.

[21] Seyed Mahdi Mazhari, Hassan Monsef, Hamid Lesani, Alireza Fereidunian, "A multi-objective 
PMU placement method considering measurement redundancy and observability value under contingencies", IEEE Trans. Power Systems, vol. 28, no. 3, pp. 2136- 2146, August, 2013.

[22] Farrokh Aminifar, A. Khodaei, M. Fotuhi-Firuzabad, M. Shahidehpour, "Contingency con-strained PMU placement in power networks", IEEE Trans. Power Systems, vol. 25, pp. 516-523, Feb, 2010.

[23] Ali Enshaee, Rahmat Allah Hooshmand, Fari-borzag Haghighatdar Fesharaki, "A new method for optimal placement of phasor measurement units to maintain full network observability under various contingencies", Elect. Power Systems Research, vol. 89, pp. 1- 10, 2012.

[24] Arash mahari, Heresh Seyedi, "Optimal PMU placement for power system observability using BICA, considering measurement redundancy", Elect. Power System Research, vol. 103, pp. 78-85, 2013.

[25] Nikolaos C. Koutsoukis, Nikolaos M. Manousakis, Pavlos S. Georgilakis, George N. Korres, "Numerical observability method for optimal phasor measurement units placement using recursive tabu search method", IET Generation, Trans \& Dist, vol. 7, pp. 110, 2013.

[26] http://www.pserc.cornell.edu/matpower.

[27] Yang, X. S. Nature-Inspired Metaheuristic Algorithms, 1st edition. Lunver Press, UK, 2008.

[28] T. L. Baldwin, L. Mili, M. B. Boisen, Jr., and R. Adapa, "Power system observability with minimal phasor measurement placement", IEEE Trans. Power Syst., vol. 8, no. 2, , pp. 707-715, May, 1993.

[29] Rajesh Kavasseri, and Sudarshan K. Srinivasan, "Joint placement of phasor and power flow measurements for observability of power systems", IEEE Trans. Power Systems, vol. 26, no. 4, pp. 1929-1936, Nov, 2011.

[30] A. Ahmadi, Y. Alinejad Beromi, M. Moradi, "Optimal PMU placement for power system observability using binary particle swarm optimization and considering measurement redundancy", Expert System Applications, vol. 38, no. 6, pp. 7263-7269, 2011.

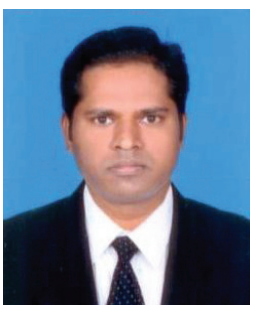

K. Arul jeyaraj pursuing $\mathrm{PhD}$ from Anna University, Chennai, India. His research area is Smart grid technology.

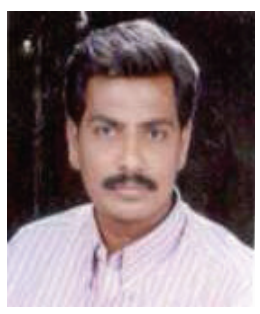

V. Rajasekaran received $\mathrm{PhD}$ degree in 2009 from M.K.U, Madurai, India. His research areas are Energy and Power Quality, etc.

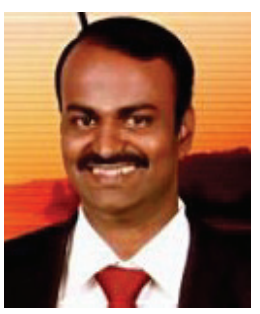

S. K. Nandha kumar received $\mathrm{PhD}$ degree in 2013 from Anna University, Chennai, India. His research area is reactive power management.

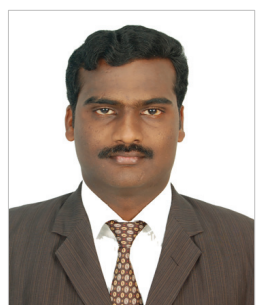

K. Chandrasekaran received $\mathrm{PhD}$ degree in 2012 from NIT, Trichy, India. His research areas are Unit commitment and Economic load dispatch. 